In der Rubrik „Literatur kompakt" werden die wichtigsten Originalarbeiten aus der internationalen Fachliteratur referiert.

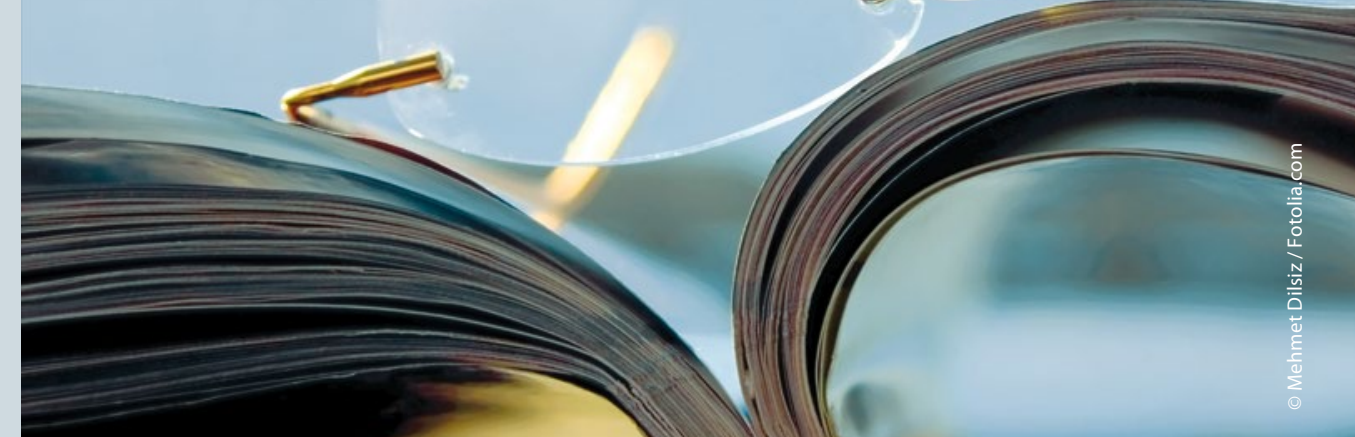

\section{Obstruktive Schlafapnoe: Zungenschrittmacher im Langzeittest}

\begin{abstract}
Mithilfe der elektrischen Hypoglossus-Nervenstimulation lässt sich die Schlafqualität von Patienten mit obstruktiver Schlafapnoe offenbar verbessern. Langzeitergebnisse fehlten aber bislang.
\end{abstract}

\begin{abstract}
7 ur CPAP-Beatmung (continous positive airway pressure), dem Goldstandard in der Behandlung der obstruktiven Schlafapnoe (OSA), gab es lange Zeit keine Alternative, bis im Jahr 2014 die ersten Daten der STAR-Studie zur atemsynchronen elektrischen Hypoglossus-Nervenstimulation mit Hilfe eines Schrittmachers veröffentlicht wurden (das Verfahren wurde in den HNONachrichten 5/2015 ausführlich beschrieben). Im Rahmen dieser prospektiven industriegesponserten Kohortenstudie hatten die Ärzte zwölf Monate nach Schrittmacherimplantation als
\end{abstract}

Endpunkt den Apnoe-Hypopnoe-Index (AHI = Anzahl der Apnoen/Hypopnoen pro Stunde) und den Sauerstoffsättigungsindex (ODI) gemessen sowie als sekundäre Endpunkte die Lebensqualität (FOSQ) und Schläfrigkeit (ESS) bestimmt.

Das haben sie nun drei Jahre nach Implantation wiederholt, wobei von den ursprünglich 126 Teilnehmern noch 116 befragt werden konnten und sich nur 98 erneut einer Polysomnografie (PSG) unterzogen haben. Die Probanden waren überwiegend Männer (83\%), im Durchschnitt 55 Jahre alt mit einem mittleren
Body-Mass-Index (BMI) von $28,4 \mathrm{~kg} / \mathrm{m}^{2}$. Ein BMI $>32 \mathrm{~kg} / \mathrm{m}^{2}$ galt als Ausschlusskriterium.

Drei Jahre nach Implantation gaben $81 \%$ der Probanden an, den Schrittmacher jede Nacht zu nutzen. In der PSGGruppe sank der AHI im Median von anfangs 28,2 $(30,4 \pm 10,4)$ Ereignissen pro Stunde auf 8,7 $(13,5 \pm 14,3)$ Ereignisse pro Stunde nach einem Jahr und blieb auch nach drei Jahren mit 6,2 (11,5 \pm 13,9) Ereignissen pro Stunde stabil. Entsprechend sank auch der ODI im zeitlichen Verlauf von median 24,3 (27,1 \pm $10,8)$ zu Beginn auf 7,1 $(12,0 \pm 13,6)$ nach zwölf Monaten und 4,8 $(9,1 \pm 11,7)$ nach drei Jahren. Neben diesen objektiv messbaren Verbesserungen fühlten sich die Teilnehmer tagsüber weniger schläfrig (ESS-Score $\leq 10: 33 \%$ zu Beginn vs. $77 \%$ nach drei Jahren), ihre Lebensqualität stieg (FOSQ-Score > 17,9: $15 \% \mathrm{zu} \mathrm{Be}$ ginn vs. $63 \%$ nach drei Jahren). Schwere unerwünschte Nebenwirkungen wurden nicht beobachtet.

Fazit: Nach Ansicht der Studienautoren ist die Hypoglossus-Stimulation auch langfristig eine erfolgreiche und adäquate Behandlungsoption für Patienten mit moderater bis schwerer OSA. Als Schwachpunkte räumen sie die fehlende Kontrollgruppe sowie einen möglichen Selektionsbias bedingt durch die Entscheidung für oder gegen eine PSG ein. Auch die Exklusion deutlich übergewichtiger Patienten könnte das Ergebnis verzerrt haben.

Dagmar Kraus

Die Implantation eines Schrittmachers zur selektiven Stimulation des N. hypoglossus kann Patienten mit OSA helfen, welche die CPAP-Beatmung nicht tolerieren oder bei denen sie versagt. 